\title{
A Traditional and Complementary Medicine (T\&CM) Use and Its Association with Diabetic Control among Diabetic Patient in Single- Center
}

Mohd Al-Baqlish, Nurain Zainab Zainal Abidin, Nur Syahirah Abdul Rahim, Nur Tasnem Binti Rusdi, Ridzuan Said

International Islamic University Malaysia

Presenter: Nurain Zainab Zainal Abidin

Introduction: Diabetes Mellitus is a major global health concern worldwide with increasing trend as reported in the National Health Morbidity Survey 2015. Due to the fact that diabetes mellitus is a life long chronic illness and incurable, it contributes to the increased tendency of seeking Traditional Complementary Medicine (TCM). The objective of this study is to measure the prevalence of CM use among diabetic patients and its association with diabetic control. Materials and Method: A cross-sectional study was performed at Hospital Tengku Ampuan Afzan, between July to August 2018. 136 Type II adult diabetic patients in medical ward and diabetic clinic were interviewed regarding the use of $C M$, and their blood investigations results were collected from patients' medical records. A data collection form was used as the study instrument. Descriptive statistic was used to measure the prevalence while Fisher exact test and Independent t-test were used to find associations between CM usage with study background and diabetic control. Results: Low prevalence of Traditional Complementary Medicine use (16.9\%) was found with retired group of respondents was identified to be the predominant group $(p$-value $=0.025)$. Majority of patients used $C M$ as an additional treatment for diabetes $(60.9 \%)$. Friends, family members and advertisement were the main influential factors in starting and using CM. A significant difference in eGFR value was reported between inpatient and outpatient CM users with a p-value of 0.001 . No positive association was observed for HbA1c level. Conclusion: 1 in every 6 diabetic patients in HTAA, Kuantan were using Traditional Complementary Medicine along with conventional diabetic medications in which retirees were the major group identified. eGFR was the diabetic control parameter that had been found to have a significant association with diabetic inpatients and outpatient CM users in this study. 